\title{
ON THE DENSITY TYPE TOPOLOGIES IN HIGHER DIMENSIONS
}

\author{
GRAŻYNA HORBACZEWSKA
}

(Received 12 April 2010)

\begin{abstract}
The topologies of the density type in Euclidean space of dimension higher than one are introduced. Definitions are based on a notion of density point connected with a set of sequences of real numbers. Our purpose is to study properties of these topologies and connections between them.
\end{abstract}

2000 Mathematics subject classification: primary 54A10.

Keywords and phrases: density points, comparison of topologies.

Following an observation that the notion of a density point (see [6]) of a measurable subset of the real line can be described by using a fixed sequence $\{n\}_{n \in \mathbb{N}}$, Filipczak and Hejduk [1] introduced the notion of a density point of a measurable subset of the real line with respect to a fixed unbounded and nondecreasing sequence of positive reals. They proved that this notion coincides with that of a classical density point if and only if the sequence in question tends to infinity not too fast.

We wish to investigate a similar notion, but on the plane and in Euclidean space of dimension higher than two, where, even in the classical case, the situation is more complicated (see [5, 6]). We shall use differentiation bases consisting of intervals of a special type.

We begin by recalling some basic definitions. Let $\mathcal{L}_{2}$ stand for the family of all Lebesgue measurable sets on the plane and let $\lambda_{2}$ stand for two-dimensional Lebesgue measure.

We say that a point $\left(x_{0}, y_{0}\right) \in \mathbb{R}^{2}$ is an ordinary density point of the set $A \in \mathcal{L}_{2}$ if and only if

$$
\lim _{h \rightarrow 0^{+}} \frac{\lambda_{2}\left(A \cap\left(\left[x_{0}-h, x_{0}+h\right] \times\left[y_{0}-h, y_{0}+h\right]\right)\right)}{4 h^{2}}=1 .
$$

We say that a point $\left(x_{0}, y_{0}\right) \in \mathbb{R}^{2}$ is a strong density point of the set $A \in \mathcal{L}_{2}$ if and only if

$$
\lim _{h \rightarrow 0^{+}, k \rightarrow 0^{+}} \frac{\lambda_{2}\left(A \cap\left(\left[x_{0}-h, x_{0}+h\right] \times\left[y_{0}-k, y_{0}+k\right]\right)\right)}{4 h k}=1 .
$$

(C) 2010 Australian Mathematical Publishing Association Inc. 0004-9727/2010 \$16.00 
Obviously if $\left(x_{0}, y_{0}\right)$ is a strong density point of $A$ then it is also an ordinary density point of $A$, but the converse need not be true.

As usual, let $\Phi_{0}(A)$ denote the set of all ordinary density points of a set $A \in \mathcal{L}_{2}$ and let $\Phi_{s}(A)$ denote the set of all strong density points of $A \in \mathcal{L}_{2}$.

For brevity, let $R((x, y), a, b)$ stand for the rectangle $(x-a, x+a) \times(y-b, y+b)$, where $x, y \in \mathbb{R}, a, b \in \mathbb{R}_{+}$, and $S((x, y), a):=R((x, y), a, a)$.

Let $\mathcal{S}$ be the family of all unbounded and nondecreasing sequences of positive reals. Sequences $\left\{s_{n}\right\}_{n \in \mathbb{N}} \in \mathcal{S}$ are denoted by $\langle s\rangle$. We divide $\mathcal{S}$ into two sets:

$$
\mathcal{S}_{0}:=\left\{\langle s\rangle \in \mathcal{S}: \liminf _{n \rightarrow \infty} \frac{s_{n}}{s_{n+1}}=0\right\}
$$

and

$$
\mathcal{S}_{+}:=\mathcal{S} \backslash \mathcal{S}_{0}=\left\{\langle s\rangle \in \mathcal{S}: \liminf _{n \rightarrow \infty} \frac{s_{n}}{s_{n+1}}>0\right\}
$$

Definition 1. Let $\langle s\rangle,\langle t\rangle \in \mathcal{S}$. For $A \in \mathcal{L}_{2}$ we define an operator

$$
\Phi_{\langle s\rangle\langle t\rangle}(A)=\left\{(x, y) \in \mathbb{R}^{2}: \lim _{n \rightarrow+\infty} \frac{\lambda_{2}\left(A \cap R\left((x, y), 1 / s_{n}, 1 / t_{n}\right)\right)}{4 / s_{n} t_{n}}=1\right\} .
$$

It is well known (see [6]) that the fact that $(x, y)$ belongs to $\Phi_{0}(A)$ is equivalent to the fact that

$$
\lim _{n \rightarrow \infty} \frac{\lambda_{2}\left(A \cap R\left((x, y), h_{n}, k_{n}\right)\right)}{4 h_{n} k_{n}}=1
$$

for each pair of sequences of positive numbers $\left\{h_{n}\right\}_{n \in \mathbb{N}},\left\{k_{n}\right\}_{n \in \mathbb{N}}$ tending to 0 and for which there exists a number $\alpha \in(0,1)$ (called the parameter of regularity) such that $\alpha<h_{n} k_{n}^{-1}<\alpha^{-1}$ for each $n \in \mathbb{N}$.

With the latter we introduce a relation between sequences from $\mathcal{S}$.

Let $\langle s\rangle,\langle t\rangle \in \mathcal{S}$. We say that $\langle s\rangle$ is regular to $\langle t\rangle$ (written $\langle s\rangle$ reg $\langle t\rangle$ ) if there exists a number $\alpha \in(0,1)$ such that $\alpha<s_{n} t_{n}^{-1}<\alpha^{-1}$ for each $n \in \mathbb{N}$.

Here are some elementary properties of this relation.

PROPERTY 2. The relation reg is an equivalence relation in $\mathcal{S}$.

PROPERTY 3. If $\langle s\rangle \in \mathcal{S}_{+}$and $\langle t\rangle \in \mathcal{S}_{0}$ then $\langle s\rangle$ cannot be regular to $\langle t\rangle$.

PROPERTY 4. Let $\langle s\rangle,\langle t\rangle \in \mathcal{S},\langle s\rangle \operatorname{reg}\langle t\rangle$ and put $k_{n}=\max \left(s_{n}, t_{n}\right)$ for $n \in \mathbb{N}$. Then $\langle k\rangle \operatorname{reg}\langle s\rangle$.

Just from the definitions and the condition equivalent to the definition of an ordinary density we get the following proposition.

Proposition 5. For any $A \in \mathcal{L}_{2}$,

$$
\bigcap_{\substack{\langle s\rangle,\langle t\rangle \in \mathcal{S} \\\langle s\rangle \operatorname{reg}\langle t\rangle}} \Phi_{\langle s\rangle\langle t\rangle}(A)=\Phi_{0}(A)
$$


Corollary 6. For any $A \in \mathcal{L}_{2}$ and for any $\langle s\rangle,\langle t\rangle \in \mathcal{S}$ such that $\langle s\rangle$ reg $\langle t\rangle$,

$$
\Phi_{0}(A) \subset \Phi_{\langle s\rangle\langle t\rangle}(A) .
$$

Let $\langle n\rangle$ denote the increasing sequence of all natural numbers.

Proposition 7. For any $A \in \mathcal{L}_{2}$,

$$
\Phi_{\langle n\rangle\langle n\rangle}(A) \subset \Phi_{0}(A) .
$$

Proof. Let $A \in \mathcal{L}_{2}$ and $(x, y) \in \Phi_{\langle n\rangle\langle n\rangle}(A)$, that is,

$$
\lim _{n \rightarrow+\infty} \frac{\lambda_{2}(A \cap S((x, y), 1 / n))}{4 / n^{2}}=1 .
$$

Let $\left\{h_{n}\right\}_{n \in \mathbb{N}}$ be a nonincreasing sequence tending to 0 . Set

$$
\underline{h}_{n}=\max \left\{\frac{1}{k}: k \in \mathbb{N} \wedge \frac{1}{k} \leq h_{n}\right\} \quad \text { and } \quad \bar{h}_{n}=\min \left\{\frac{1}{k}: k \in \mathbb{N} \wedge \frac{1}{k} \geq h_{n}\right\}
$$

for $n \in \mathbb{N}$. Then the quotients $\underline{h}_{n} / h_{n}$ and $\bar{h}_{n} / h_{n}$ tend to 1 . Since

$$
\frac{\underline{h}_{n}^{2}}{h_{n}^{2}} \frac{\lambda_{2}\left(A \cap S\left((x, y), \underline{h}_{n}\right)\right)}{4 \underline{h}_{n}^{2}} \leq \frac{\lambda_{2}\left(A \cap S\left((x, y), h_{n}\right)\right)}{4 h_{n}^{2}} \leq \frac{\bar{h}_{n}^{2}}{h_{n}^{2}} \frac{\lambda_{2}\left(A \cap S\left((x, y) \bar{h}_{n}\right)\right)}{4 \bar{h}_{n}^{2}}
$$

Equation (1) gives

$$
\lim _{h \rightarrow 0^{+}} \frac{\lambda_{2}(A \cap S((x, y), h))}{4 h^{2}}=1 .
$$

Corollary 8. For any $A \in \mathcal{L}_{2}$,

$$
\Phi_{0}(A)=\Phi_{\langle n\rangle\langle n\rangle}(A) .
$$

Proposition 9. For every $\langle s\rangle \in \mathcal{S}_{+},\langle u\rangle \in \mathcal{S}$ and for every $A \in \mathcal{L}_{2}$,

$$
\Phi_{\langle s\rangle\langle s\rangle}(A) \subset \Phi_{\langle u\rangle\langle u\rangle}(A) .
$$

Proof. Let $\langle s\rangle \in \mathcal{S}_{+},\langle u\rangle \in \mathcal{S}, A \in \mathcal{L}_{2}$ and $(x, y) \in \Phi_{\langle s\rangle\langle s\rangle}(A)$. Denoting the complement of $A$ by $B$, we assert that $\lim _{n \rightarrow+\infty} \lambda_{2}\left(B \cap S\left((x, y), 1 / s_{n}\right)\right) /\left(4 / s_{n}^{2}\right)=0$ and $\liminf _{n \rightarrow+\infty} s_{n} / s_{n+1}=g>0$.

Let $\epsilon>0$. There exists $n_{0} \in \mathbb{N}$ such that for every $n \in \mathbb{N}, n>n_{0}$, we get

$$
\frac{\lambda_{2}\left(B \cap S\left((x, y), 1 / s_{n}\right)\right)}{4 / s_{n}^{2}}<\epsilon \cdot \frac{g^{2}}{4} \text { and } \frac{s_{n}}{s_{n+1}}>\frac{g^{2}}{4} .
$$


There exists $k_{0} \in \mathbb{N}$ such that $s_{n_{o}} \leq u_{k_{0}}$. Fix $k \in \mathbb{N}, k>k_{0}$. There exists $n \in \mathbb{N}, n \geq n_{0}$, such that $s_{n} \leq u_{k} \leq s_{n+1}$. Thus

$$
\begin{aligned}
\frac{\lambda_{2}\left(B \cap S\left((x, y), 1 / u_{k}\right)\right)}{4 / u_{k}^{2}} & \leq \frac{\lambda_{2}\left(B \cap S\left((x, y), 1 / s_{n}\right)\right)}{4 /\left(s_{n+1}\right)^{2}} \\
& =\frac{\lambda_{2}\left(B \cap S\left((x, y), 1 / s_{n}\right)\right)}{4 / s_{n}^{2}} \cdot\left(\frac{s_{n+1}}{s_{n}}\right)^{2}<\epsilon \cdot \frac{g^{2}}{4} \cdot \frac{4}{g^{2}}=\epsilon,
\end{aligned}
$$

so $(x, y) \in \Phi_{\langle u\rangle\langle u\rangle}(A)$.

COROllary 10. For every $\langle s\rangle \in \mathcal{S}_{+},\langle u\rangle \in \mathcal{S}_{0}$ and for every $A \in \mathcal{L}_{2}$,

$$
\Phi_{\langle s\rangle\langle s\rangle}(A)=\Phi_{0}(A) \subset \Phi_{\langle u\rangle\langle u\rangle}(A) .
$$

COROllary 11. For every $A \in \mathcal{L}_{2}$,

$$
\bigcap_{\langle s\rangle \in \mathcal{S}} \Phi_{\langle s\rangle\langle s\rangle}(A)=\Phi_{0}(A) .
$$

Proposition 12. Let $\langle s\rangle \in \mathcal{S}$. If $\Phi_{\langle s\rangle\langle s\rangle}(A)=\Phi_{0}(A)$ for every $A \in \mathcal{L}_{2}$ then $\langle s\rangle \in \mathcal{S}_{+}$.

PRoOF. Let $\langle s\rangle \in \mathcal{S}_{0}$. By [3, Theorem 3] there exists $Y \subset \mathbb{R}$ such that 0 is not a density point of $Y$ and

$$
\lim _{n \rightarrow+\infty} \frac{\lambda_{1}\left(Y \cap\left(-1 / s_{n}, 1 / s_{n}\right)\right)}{2 / s_{n}}=1 .
$$

Define

$$
A:=\bigcup_{y \in Y \cap[0 ;+\infty)}((\{-y, y\} \times[-y, y]) \cup([-y, y] \times\{-y, y\})) .
$$

By [3, Corollary 2.7] the set $A$ cannot have 0 as its ordinary density point on the plane.

Analysis similar to that in [3, proof of Theorem 2.6] shows that $(0,0) \in$ $\Phi_{\langle s\rangle\langle s\rangle}(A)$.

Summarizing, we have the following theorem.

THeorem 13. Let $\langle s\rangle \in \mathcal{S}$. The set of ordinary density points of $A$ is equal to $\Phi_{\langle s\rangle\langle s\rangle}(A)$ for every $A \in \mathcal{L}_{2}$ if and only if the sequence $\langle s\rangle$ belongs to $\mathcal{S}_{+}$.

We are led to the following stronger version of Corollary 11.

Proposition 14. For every $A \in \mathcal{L}_{2}$,

$$
\bigcap_{\langle s\rangle \in \mathcal{S}_{0}} \Phi_{\langle s\rangle\langle s\rangle}(A)=\Phi_{0}(A) .
$$


PROOF. One inclusion comes from Corollary 10.

To show the second one, suppose that there exists a point $(x, y) \in$ $\bigcap_{\langle s\rangle \in \mathcal{S}_{0}} \Phi_{\langle s\rangle\langle s\rangle}(A) \backslash \Phi_{0}(A)$. Then

$$
\liminf _{h \rightarrow 0^{+}} \frac{\lambda_{2}(A \cap S((x, y), h))}{4 h^{2}}<1,
$$

hence there exists a sequence $\langle s\rangle \in \mathcal{S}$ such that

$$
\lim _{n \rightarrow+\infty} \frac{\lambda_{2}\left(A \cap S\left((x, y), 1 / s_{n}\right)\right)}{4 / s_{n}^{2}}<1 .
$$

We choose a subsequence $\langle t\rangle \subset\langle s\rangle$ such that $\langle t\rangle \in \mathcal{S}_{0}$. Thus

$$
\lim _{n \rightarrow+\infty} \frac{\lambda_{2}\left(A \cap S\left((x, y), 1 / t_{n}\right)\right)}{4 / t_{n}^{2}}<1,
$$

so $(x, y) \notin \Phi_{\langle s\rangle\langle s\rangle}(A)$, which is a contradiction.

We wish to investigate whether we obtain something different by considering rectangles described by sequences.

Proposition 15. For every $\langle s\rangle,\langle t\rangle \in \mathcal{S}_{+}$such that $\langle s\rangle \operatorname{reg}\langle t\rangle, \Phi_{\langle s\rangle\langle t\rangle}(A) \subset \Phi_{0}(A)$ for every $A \in \mathcal{L}_{2}$.

PRoOF. Let $A \in \mathcal{L}_{2},\langle s\rangle,\langle t\rangle \in \mathcal{S}_{+},\langle s\rangle \operatorname{reg}\langle t\rangle$. Put $k_{n}=\max \left(s_{n}, t_{n}\right)$ for every $n \in \mathbb{N}$. It suffices to prove that $\Phi_{\langle s\rangle\langle t\rangle}(A) \subset \Phi_{\langle k\rangle\langle k\rangle}(A)$, since Properties 4 and 3 show that $\langle k\rangle \in \mathcal{S}_{+}$and $\Phi_{\langle k\rangle\langle k\rangle}(A)=\Phi_{0}(A)$, by Theorem 13 .

Suppose that there exists a point $(x, y) \in \Phi_{\langle s\rangle\langle t\rangle}(A) \backslash \Phi_{\langle k\rangle\langle k\rangle}(A)$. Let $\mathcal{B}:=\mathbb{R}^{2} \backslash A$. Then

$$
\limsup _{n \rightarrow+\infty} \frac{\lambda_{2}\left(B \cap S\left((x, y), 1 / k_{n}\right)\right)}{4 / k_{n}^{2}}>0,
$$

so there exist $\gamma>0$ and a subsequence $\left\{k_{n_{l}}\right\}_{l \in \mathbb{N}}$ of $\left\{k_{n}\right\}$ such that

$$
\lim _{l \rightarrow+\infty} \frac{\lambda_{2}\left(B \cap S\left((x, y), 1 / k_{n_{l}}\right)\right)}{4 / k_{n_{l}}^{2}}=\gamma .
$$

From this there exists $l_{0} \in \mathbb{N}$ such that for every $l \in \mathbb{N}, l>l_{0}$,

$$
\frac{\lambda_{2}\left(B \cap S\left((x, y), 1 / k_{n_{l}}\right)\right)}{4 / k_{n_{l}}^{2}}>\frac{\gamma}{2} .
$$

Hence

$$
\frac{\lambda_{2}\left(B \cap R\left((x, y), 1 / s_{n_{l}}, 1 / t_{n_{l}}\right)\right)}{4 /\left(s_{n_{l}} t_{n_{l}}\right)} \geq \frac{\lambda_{2}\left(B \cap S\left((x, y), 1 / k_{n_{l}}\right)\right)}{4 / k_{n_{l}}^{2}} \cdot \frac{t_{n_{l}}}{k_{n_{l}}} \cdot \frac{s_{n_{l}}}{k_{n_{l}}}>\frac{\gamma}{2} \lambda^{2}>0,
$$

and $(x, y) \notin \Phi_{\langle s\rangle\langle t\rangle}(A)$, which is a contradiction. 
COROLlary 16. If $\langle s\rangle,\langle t\rangle \in \mathcal{S}_{+}$and $\langle s\rangle \operatorname{reg}\langle t\rangle$, then $\Phi_{0}(A)=\Phi_{\langle s\rangle\langle t\rangle}(A)$ for every $A \in \mathcal{L}_{2}$.

Proposition 17. Let $\langle s\rangle,\langle t\rangle \in \mathcal{S}_{+}$or $\langle s\rangle,\langle t\rangle \in \mathcal{S}_{0}$. If $\Phi_{\langle s\rangle\langle t\rangle}(A)=\Phi_{0}(A)$ for every $A \in \mathcal{L}_{2}$, then $\langle s\rangle \operatorname{reg}\langle t\rangle$.

PROOF. Let $\langle s\rangle,\langle t\rangle \in \mathcal{S}_{+}$or $\langle s\rangle,\langle t\rangle \in \mathcal{S}_{0}$. Assume that $\langle s\rangle$ reg $\langle t\rangle$ fails, that is, for every $\alpha \in(0,1)$ there exists $n \in \mathbb{N}$ such that $\left(s_{n} / t_{n}\right) \leq \alpha$ or $\left(s_{n} / t_{n}\right) \geq(1 / \alpha)$. Therefore there exists a subsequence $\left\{n_{k}\right\}_{k \in \mathbb{N}}$ such that $\left\{s_{n_{k}} / t_{n_{k}}\right\}_{k \in \mathbb{N}}$ tends monotonically to $+\infty$ or to zero. We will assume that the second case holds, for the first case is analogous. We will assume additionally, by choosing a subsequence if necessary, that $s_{n_{k+1}}>2 s_{n_{k}}$.

Define a function $f:\left(0,1 / 2 s_{n_{1}}\right] \rightarrow \mathbb{R}$, where $f\left(1 / 2 s_{n_{k}}\right)=1 / t_{n_{k}}$ for $n \in \mathbb{N}$ and $f$ is linear and continuous on the intervals $\left[1 / 2 s_{n_{k+1}}, 1 / 2 s_{n_{k}}\right], k \in \mathbb{N}$.

Since for every $x \in\left(1 / 2 s_{n_{k+1}}, 1 / 2 s_{n_{k}}\right)$ the quotient $f(x) / x$ is between

$$
\frac{f\left(1 / 2 s_{n_{k}}\right)}{1 / 2 s_{n_{k}}}=\frac{2 s_{n_{k}}}{t_{n_{k}}} \quad \text { and } \quad \frac{f\left(1 / 2 s_{n_{k+1}}\right)}{1 / 2 s_{n_{k+1}}}=\frac{2 s_{n_{k+1}}}{t_{n_{k+1}}},
$$

it follows that

$$
\lim _{x \rightarrow 0^{+}} \frac{f(x)}{x}=0
$$

Set

$$
A=[-1,1]^{2} \backslash\left\{(x, y): x \in\left(0,1 / 2 s_{n_{1}}\right) \wedge y \in(0, f(x))\right\}
$$

Then

$$
\frac{\lambda_{2}\left(A \cap[-h, h]^{2}\right)}{4 h^{2}} \geq \frac{3 h^{2}+h(h-f(h))}{4 h^{2}}=1-\frac{f(h)}{4 h} \rightarrow 1
$$

for $h \rightarrow 0^{+}$, so $(0,0) \in \Phi_{0}(A)$. However,

$$
\lambda_{2}\left(\left(\mathbb{R}^{2} \backslash A\right) \cap R\left((0,0), 1 / s_{n_{k}}, 1 / t_{n_{k}}\right)\right) \geq \frac{1}{2} \cdot \frac{1}{s_{n_{k}}} \cdot \frac{1}{t_{n_{k}}},
$$

therefore $(0,0) \notin \Phi_{\langle s\rangle\langle t\rangle}(A)$. Thus we have found a set $A$ for which $\Phi_{\langle s\rangle\langle t\rangle}(A) \neq$ $\Phi_{0}(A)$.

Summarizing Corollary 16 and Proposition 17, we have the following theorem.

THEOREM 18. Let $\langle s\rangle,\langle t\rangle \in \mathcal{S}_{+}$. The set of ordinary density points of $A$ is equal to $\Phi_{\langle s\rangle\langle t\rangle}(A)$ for every $A \in \mathcal{L}_{2}$ if and only if $\langle s\rangle$ reg $\langle t\rangle$.

Proposition 19. For every $A \in \mathcal{L}_{2}$,

$$
\bigcap_{\langle s\rangle,\langle t\rangle \in \mathcal{S}} \Phi_{\langle s\rangle\langle t\rangle}(A)=\Phi_{s}(A) .
$$


Proof. Let $A \in \mathcal{L}_{2}$. By the Heine definition of limit, each point of strong density of $A$ belongs to $\Phi_{\langle s\rangle\langle t\rangle}(A)$ for every $\langle s\rangle,\langle t\rangle \in \mathcal{S}$.

Suppose that there exists a point $(x, y)$ belonging to $\bigcap_{\langle s\rangle,\langle t\rangle \in \mathcal{S}} \Phi_{\langle s\rangle\langle t\rangle}(A)$ but which is not a strong density point of $A$. Hence there exist decreasing sequences $\left\{k_{n}\right\}_{n \in \mathbb{N}}$ and $\left\{h_{n}\right\}_{n \in \mathbb{N}}$ tending to 0 such that

$$
\lim _{n \rightarrow+\infty} \frac{\lambda_{2}\left(A \cap R\left((x, y), h_{n}, k_{n}\right)\right)}{4 h_{n} k_{n}}<1 .
$$

Then for $s_{n}=1 / h_{n}, t_{n}=1 / k_{n}, n \in \mathbb{N}$, we obtain $(x, y) \notin \Phi_{\langle s\rangle\langle t\rangle}(A)$, which is a contradiction.

Proposition 20. For every $\langle s\rangle \in \mathcal{S}$ there exists $\langle t\rangle \in \mathcal{S}$ which is not regular to $\langle s\rangle$ and there exists a set $A \in \mathcal{L}_{2}$ such that the difference $\Phi_{\langle s\rangle\langle s\rangle}(A) \backslash \Phi_{\langle s\rangle\langle t\rangle}(A)$ is nonempty.

Proof. Let $\langle s\rangle \in \mathcal{S}$. Define $t_{n}:=s_{n}^{2}$ for $n \in \mathbb{N}$. Then $\langle t\rangle$ is not regular to $\langle s\rangle$. Set $A:=\left\{(x, y): y>x^{2} \vee y<-x^{2}\right\}$. It is clear that

$$
(0,0) \in \Phi(A) \subset \Phi_{\langle s\rangle\langle s\rangle}(A) \quad \text { and } \quad \frac{\lambda_{2}\left(A \cap R\left((0,0), 1 / s_{n}, 1 / s_{n}^{2}\right)\right)}{4 / s_{n}^{3}}=\frac{2}{3},
$$

so $(0,0) \notin \Phi_{\langle s\rangle\langle t\rangle}(A)$.

Proposition 21. For every $\langle s\rangle \in \mathcal{S}$ there exists $\langle t\rangle \in \mathcal{S}$ which is not regular to $\langle s\rangle$ and there exists a set $A \in \mathcal{L}_{2}$ such that the difference $\Phi_{\langle s\rangle\langle t\rangle}(A) \backslash \Phi_{0}(A)$ is nonempty.

Proof. Let $\langle s\rangle \in \mathcal{S}$. Define $t_{n}:=n \cdot s_{n}$ for $n \in \mathbb{N}$. Then $\langle t\rangle$ is not regular to $\langle s\rangle$. Moreover, $\langle t\rangle \in \mathcal{S}_{0}$ if and only if $\langle s\rangle \in \mathcal{S}_{0}$. We choose a subsequence $\left\{t_{n_{k}}\right\}_{k \in \mathbb{N}} \subset$ $\left\{t_{n}\right\}_{n \in \mathbb{N}}$ such that $t_{n_{k+1}}>2 t_{n_{k}}$. Set

$$
A:=[-1,1]^{2} \backslash \bigcup_{k \in \mathbb{N}}\left[1 / 2 t_{n_{k}}, 1 / t_{t_{n_{k}}}\right]^{2}
$$

and denote by $B$ the complement of the set $A$.

We now consider two cases. If $1 / t_{n} \in\left(1 / 2 t_{n_{k}}, 1 / t_{n_{k}}\right)$ then

$$
\begin{aligned}
\lambda_{2}\left(B \cap R\left((0,0), \frac{1}{s_{n}}, \frac{1}{t_{n}}\right)\right) \leq & \frac{1}{t_{n}} \cdot \frac{1}{2 t_{n_{k}}}+\left(\frac{1}{t_{n}}-\frac{1}{2 t_{n_{k}}}\right)^{2} \\
& +\left(\frac{1}{t_{n_{k}}}-\frac{1}{t_{n_{k}}}\right)\left(\frac{1}{t_{n}}-\frac{1}{2 t_{n_{k}}}\right) \\
& \leq \frac{1}{t_{n}} \cdot \frac{1}{2 t_{n_{k}}}+\left(\frac{1}{t_{n}}-\frac{1}{2 t_{n_{k}}}\right)^{2}+\frac{1}{2 t_{n_{k}}}\left(\frac{1}{t_{n}}-\frac{1}{2 t_{n_{k}}}\right) \\
& \left(\frac{1}{t_{n}}\right)^{2} .
\end{aligned}
$$

If $1 / t_{n} \in\left(1 / t_{n_{k+1}}, 1 / 2 t_{n_{k}}\right)$ then $B \cap R\left((0,0), 1 / s_{n}, 1 / t_{n}\right) \subset\left[0,1 / t_{n}\right]^{2}$. 
Therefore

$$
\frac{\lambda_{2}\left(B \cap R\left((0,0), 1 / s_{n}, 1 / t_{n}\right)\right)}{4 / s_{n} t_{n}} \leq \frac{\left(1 / t_{n}\right)^{2}}{4 / s_{n} t_{n}}=\frac{1}{4 n},
$$

so $(0,0) \in \Phi_{\langle s\rangle\langle t\rangle}(A)$.

The point $(0,0)$ is clearly not an ordinary density point of $A$ since

$$
\lambda_{2}\left(B \cap S\left((0,0), 1 / t_{n_{k}}\right)\right) \geq \frac{1}{4}\left(1 / t_{n_{k}}\right)^{2} .
$$

This concludes the proof.

After a slight modification of the last proof we can obtain the following proposition.

Proposition 22. For every $\langle s\rangle \in \mathcal{S}_{0}$ there exists $\langle t\rangle \in \mathcal{S}$, which is not regular to $\langle s\rangle$, and there exists a set $A \in \mathcal{L}_{2}$ such that the difference $\Phi_{\langle s\rangle\langle t\rangle}(A) \backslash \Phi_{\langle s\rangle\langle s\rangle}(A)$ is nonempty.

PRoposition 23. For every $\langle s\rangle \in \mathcal{S}_{0}$ there exists $\langle t\rangle \in \mathcal{S}$, such that $\langle s\rangle$ reg $\langle t\rangle$ and there exists a set $A \in \mathcal{L}_{2}$ such that the difference $\Phi_{\langle s\rangle\langle s\rangle}(A) \backslash \Phi_{\langle s\rangle\langle t\rangle}(A)$ is nonempty.

PRoOf. Let $\langle s\rangle \in \mathcal{S}_{0}$. Define $t_{n}:=2 s_{n}$ for $n \in \mathbb{N}$. Then $\langle s\rangle$ reg $\langle t\rangle$. Let $\left\{s_{n_{k}}\right\}_{k \in \mathbb{N}}$ be a subsequence of $\langle s\rangle$ such that

$$
\lim _{k \rightarrow+\infty} \frac{s_{n_{k}}}{s_{n_{k}+1}}=0 .
$$

Set

$$
B:=\bigcup_{k \in \mathbb{N}}\left(\left(-\frac{1}{s_{n_{k}+1}}, \frac{1}{s_{n_{k}+1}}\right] \times\left(\left[-\frac{2}{s_{n_{k}+1}},-\frac{1}{s_{n_{k}+1}}\right] \cup\left[\frac{1}{s_{n_{k}+1}}, \frac{2}{s_{n_{k}+1}}\right]\right)\right) .
$$

Let $\epsilon>0$. There exists $k_{0} \in \mathbb{N}$, such that for any $k>k_{0}$ we have $s_{n_{k}} / s_{n_{k}+1}<\sqrt{\epsilon / 2}$. Set $k(n):=\min \left\{k \in \mathbb{N}: s_{n_{k}} \geq s_{n}\right\}$ and choose $n_{0}$ for which $k\left(n_{0}\right)>k_{0}$. Then for every $n>n_{0}$,

$$
\frac{\lambda_{2}\left(B \cap S\left((0,0), 1 / s_{n}\right)\right)}{4 / s_{n}^{2}} \leq \frac{8\left(1 / s_{n_{k(n)}+1}\right)^{2}}{4\left(1 / s_{n_{k(n)}}\right)^{2}}=2\left(\frac{s_{n_{k(n)}}}{s_{n_{k(n)}+1}}\right)^{2}<\epsilon,
$$

so, denoting by $A$ the complement of $B$, we get $(0,0) \in \Phi_{\langle s\rangle\langle s\rangle}(A)$.

Since for every $k \in \mathbb{N}$,

$$
\lambda_{2}\left(B \cap R\left((0,0), 1 / s_{n_{k}+1}, 1 / t_{n_{k}+1}\right)\right) \geq 2 \cdot \frac{1}{s_{n_{k}+1}} \cdot \frac{1}{t_{n_{k}+1}},
$$

then

$$
\limsup _{n \rightarrow+\infty} \frac{\lambda_{2}\left(B \cap R\left((0,0), 1 / s_{n}, 1 / t_{n}\right)\right)}{4 / s_{n} t_{n}}>0 .
$$

Therefore $(0,0) \notin \Phi_{\langle s\rangle\langle t\rangle}(A)$.

Proposition 24. For every $\langle s\rangle \in \mathcal{S}_{0}$ there exists $\langle t\rangle \in \mathcal{S}$ such that $\langle s\rangle$ reg $\langle t\rangle$ and there exists a set $A \in \mathcal{L}_{2}$ such that $\Phi_{\langle s\rangle\langle t\rangle}(A) \backslash \Phi_{\langle s\rangle\langle s\rangle}(A) \neq \emptyset$. 
Proof. Let $\langle s\rangle \in \mathcal{S}_{0}$. Define $t_{n}:=(1 / 2) s_{n}$ for $n \in \mathbb{N}$. Then $\langle s\rangle$ reg $\langle t\rangle$. Let $\left\{s_{n_{k}}\right\}_{k \in \mathbb{N}}$ be a subsequence of $\langle s\rangle$ such that $\lim _{k \rightarrow+\infty} s_{n_{k}} / s_{n_{k}+1}=0$.

Set

$$
\mathcal{B}:=\bigcup_{k \in \mathbb{N}}\left(\left[-\frac{1}{s_{n_{k}+1}}, \frac{1}{s_{n_{k}+1}}\right] \times\left(\left[-\frac{1}{s_{n_{k}+1}},-\frac{1}{2 s_{n_{k}+1}}\right] \cup\left[\frac{1}{2 s_{n_{k}+1}}, \frac{1}{s_{n_{k}+1}}\right]\right)\right)
$$

and let $A$ be the complement of $\mathcal{B}$.

For every $k \in \mathbb{N}$,

$$
\lambda_{2}\left(B \cap S\left((0,0), 1 / s_{n_{k}+1}\right)\right)=2 / s_{n_{k}+1},
$$

hence

$$
\limsup _{n \rightarrow+\infty} \frac{\lambda_{2}\left(B \cap S\left((0,0), 1 / s_{n}\right)\right)}{4 / s_{n}^{2}}>0
$$

so $(0,0) \notin \Phi_{\langle s\rangle\langle t\rangle}(A)$.

Let $\epsilon>0$. There exists $k_{0} \in \mathbb{N}$ such that for any $k>k_{0}$ we have $\left(s_{n_{k}} / s_{n_{k}+1}\right)<\sqrt{2 \epsilon}$. Set $k(n)=\min \left\{k \in \mathbb{N}: s_{n_{k}} \geq s_{n}\right\}$ and choose $n_{0}$ for which $k\left(n_{0}\right)>k_{0}$. Then for every $n>n_{0}$,

$$
\frac{\lambda_{2}\left(B \cap R\left((0,0), 1 / s_{n}, 1 / t_{n}\right)\right)}{4 / s_{n} t_{n}}<\frac{4 \cdot\left(1 / s_{n_{k(n)}+1}\right)^{2}}{8 / s_{n}^{2}}<\frac{1}{2}\left(\frac{s_{n(k)}}{s_{n_{k(n)}+1}}\right)^{2}<\epsilon .
$$

Therefore $(0,0) \in \Phi_{\langle s\rangle\langle t\rangle}(A)$.

We have considered connections between operators $\Phi_{\langle s\rangle\langle t\rangle}$ depending on sequences $\langle s\rangle,\langle t\rangle \in \mathcal{S}$. Now let us mention general results on such operators.

Proposition 25. For every $\langle s\rangle,\langle t\rangle \in \mathcal{S}$ and for every $A \in \mathcal{L}_{2}$ the set $\Phi_{\langle s\rangle\langle t\rangle}(A)$ is an $F_{\sigma \delta}$ set.

PROOF. We first observe that

$$
\Phi_{\langle s\rangle\langle t\rangle}(A)=\bigcap_{k=1}^{\infty} \bigcup_{m=1}^{\infty} \bigcap_{n=m}^{\infty}\left\{(x, y): \frac{\lambda_{2}\left(A \cap R\left((x, y), 1 / s_{n}, 1 / t_{n}\right)\right)}{4 / s_{n} t_{n}} \geq 1-\frac{1}{k}\right\}
$$

for every $\langle s\rangle,\langle t\rangle \in \mathcal{S}$ and every $A \in \mathcal{L}_{2}$.

Since for a fixed $n$ the function

$$
\frac{\lambda_{2}\left(A \cap R\left((x, y), 1 / s_{n}, 1 / t_{n}\right)\right)}{4 / s_{n} t_{n}}
$$

is a continuous function of $(x, y)$ the set

$$
\bigcap_{n=m}^{\infty}\left\{(x, y): \frac{\lambda_{2}\left(A \cap R\left((x, y), 1 / s_{n}, 1 / t_{n}\right)\right)}{4 / s_{n} t_{n}} \geq 1-\frac{1}{k}\right\}
$$

is closed, so $\Phi_{\langle s\rangle\langle t\rangle}(A)$ is an $F_{\sigma \delta}$ set. 
THEOREM 26. For every $\langle s\rangle,\langle t\rangle \in \mathcal{S}$ and for every $A, B \in \mathcal{L}_{2}$ :

(1) $\Phi_{\langle s\rangle\langle t\rangle}(\emptyset)=\emptyset, \Phi_{\langle s\rangle\langle t\rangle}\left(\mathbb{R}^{2}\right)=\mathbb{R}^{2}$;

(2) $\Phi_{\langle s\rangle\langle t\rangle}(A \cap B)=\Phi_{\langle s\rangle\langle t\rangle}(A) \cap \Phi_{\langle s\rangle\langle\langle\rangle}(B)$;

(3) $\quad A \sim B \Longrightarrow \Phi_{\langle s\rangle\langle t\rangle}(A)=\Phi_{\langle s\rangle\langle t\rangle}(B)$;

(4) $A \sim \Phi_{\langle s\rangle\langle t\rangle}(A)$,

where $A \sim B$ means that $\lambda_{2}(A \triangle B)=0$.

ProOF. (1), (2) and (3) are obvious. (4) is a simple consequence of the inclusion $\Phi_{s}(A) \subset \Phi_{\langle s\rangle\langle t\rangle}(A)$ (Proposition 19) and the fact that the operator $\Phi_{s}$ is a lower density operator (which means it satisfies conditions (1)-(4)).

We now recall results presented in [4]. Let $(X, \mathbb{S}, I)$ denote a measurable space, where $\mathbb{S}$ is a $\sigma$-algebra of subsets of $X$ and $I$ is a proper $\sigma$-ideal of $\mathbb{S}$-measurable sets. The space $(X, \mathbb{S}, I)$ is said to have the hull property if whenever $A \subset X$, there is a set $B \in \mathbb{S}$ such that $A \subset B$ and if $Z \in \mathbb{S}$ and $A \subset Z$, then $B \backslash Z \in I$.

For every lower density operator $\Phi$ on $\mathbb{S}$ let $\mathcal{T}_{\Phi}:=\{A \in \mathbb{S}: A \subset \Phi(A)\}$.

THEOREM 27 [4]. Let $(X, \mathbb{S}, I)$ be a measurable space having the hull property. Then for every lower density operator $\Phi$ the family $\mathcal{T}_{\Phi}$ is a topology on $X$.

For every $\langle s\rangle,\langle t\rangle \in \mathcal{S}$ we define a family $\mathcal{T}_{\langle s\rangle\langle t\rangle}:=\left\{A \in \mathcal{L}_{2}: A \subset \Phi_{\langle s\rangle\langle\langle\rangle}(A)\right\}$. Since the assumptions of the last theorem are fulfilled $\mathcal{T}_{\langle s\rangle\langle t\rangle}$ is a topology on the plane.

For a deeper discussion of properties of this type of topology following from the properties of the operator, we refer the reader to [2].

We will need only one additional property of general lower density operators.

THEOREM 28. Let $\Phi_{1}, \Phi_{2}$ be lower density operators in a measurable space $(X, \mathbb{S}, I)$. Then $\mathcal{T}_{\Phi_{1}}=\mathcal{T}_{\Phi_{2}}$ if and only if $\Phi_{1}=\Phi_{2}$.

ProOF. Sufficiency is obvious.

Suppose that $\mathcal{T}_{\Phi_{1}}=\mathcal{T}_{\Phi_{2}}$ but that there exists a set $A \in \mathbb{S}$ such that $\Phi_{1}(A) \backslash$ $\Phi_{2}(A)=\emptyset$. Since $\Phi_{1}$ is a lower density operator, $\Phi_{1}(A)$ belongs to $\mathcal{T}_{\Phi_{1}}$ and, by our supposition, belongs also to $\mathcal{T}_{\Phi_{2}}$, which means that $\Phi_{1}(A) \subset \Phi_{2}\left(\Phi_{1}(A)\right.$ ), but $A \triangle \Phi_{1}(A) \in I$, so $\Phi_{2}(A)=\Phi_{2}\left(\Phi_{1}(A)\right)$. Therefore $\Phi_{1}(A) \subset \Phi_{2}(A)$, which is a contradiction.

By virtue of the above theorem and the properties shown earlier we get relations between topologies $\mathcal{T}_{\langle s\rangle\langle t\rangle},\langle s\rangle,\langle t\rangle \in \mathcal{S}$.

THEOREM 29. (1) $\bigcap_{\substack{\langle s\rangle,\langle t\rangle \in \mathcal{S} \\\langle s\rangle \operatorname{reg}\langle t\rangle}} \mathcal{T}_{\langle s\rangle\langle t\rangle}=\mathcal{T}_{0}$, where $\mathcal{T}_{0}$ denotes the ordinary density topology on the plane.

(2) $\bigcap_{\langle s\rangle \in \mathcal{S}_{0}} \mathcal{T}_{\langle s\rangle\langle s\rangle}=\mathcal{T}_{0}$.

(3) For every $\langle s\rangle \in \mathcal{S}, \mathcal{T}_{\langle s\rangle\langle s\rangle}=\mathcal{T}_{0}$ if and only if $\langle s\rangle \in \mathcal{S}_{+}$.

(4) For every $\langle s\rangle,\langle t\rangle \in \mathcal{S}_{+} \mathcal{T}_{\langle s\rangle\langle t\rangle}=\mathcal{T}_{0}$ if and only if $\langle s\rangle \operatorname{reg}\langle t\rangle$. 
(5) $\bigcap_{\langle s\rangle,\langle t\rangle \in \mathcal{S}} \mathcal{T}_{\langle s\rangle\langle t\rangle}=\mathcal{T}_{s}$, where $\mathcal{T}_{s}$ denotes the strong density topology on the plane.

(6) For every $\langle s\rangle \in \mathcal{S}$ there exist $\langle p\rangle,\langle t\rangle \in \mathcal{S}$ which are not regular to $\langle s\rangle$ and such that $\mathcal{T}_{\langle s\rangle\langle s\rangle} \backslash \mathcal{T}_{\langle s\rangle\langle t\rangle} \neq \emptyset$ and $\mathcal{T}_{\langle s\rangle\langle p\rangle} \backslash \mathcal{T}_{\langle s\rangle\langle s\rangle} \neq \emptyset$.

(7) For every $\langle s\rangle \in \mathcal{S}_{0}$ there exist $\langle p\rangle,\langle t\rangle \in \mathcal{S}$ such that $\langle t\rangle \operatorname{reg}\langle s\rangle,\langle p\rangle \operatorname{reg}\langle s\rangle$, $\mathcal{T}_{\langle s\rangle\langle s\rangle} \backslash \mathcal{T}_{\langle s\rangle\langle t\rangle} \neq \emptyset$ and $\mathcal{T}_{\langle s\rangle\langle p\rangle} \backslash \mathcal{T}_{\langle s\rangle\langle s\rangle} \neq \emptyset$.

Here are some natural properties of the $\mathcal{T}_{\langle s\rangle\langle t\rangle}$ topologies. Property (5) from the next theorem makes property (2) from the previous theorem more interesting since the topology $\mathcal{T}_{0}$ is invariant under similarity but, as we will see, $\mathcal{T}_{\langle s\rangle\langle s\rangle}$ for $\langle s\rangle \in \mathcal{S}_{0}$ is not.

We will use the following notation: for $A \in \mathbb{R}^{2}$ and $x, y \in \mathbb{R}$, write $A+(x, y)$ for $\{(a+x, b+y): a, b \in A\},-A$ for $\{(-a,-b): a, b \in A\}$ and $(x, y) \cdot A$ for $\{(x a, y b): a, b \in A\}$.

THEOREM 30. For every $A \in \mathcal{L}_{2}$ and for every $\langle s\rangle,\langle t\rangle \in \mathcal{S}$ :

(1) for every $x, y \in \mathbb{R}$, if $A \in \mathcal{T}_{\langle s\rangle\langle t\rangle}$ then $A+(x, y) \in \mathcal{T}_{\langle s\rangle\langle t\rangle}$;

(2) if $A \in \mathcal{T}_{\langle s\rangle\langle t\rangle}$ then $-A \in \mathcal{T}_{\langle s\rangle\langle t\rangle}$;

(3) for every $m, p \in \mathbb{R}$, such that $|m| \geq 1$ and $|p| \geq 1$, if $A \in \mathcal{T}_{\langle s\rangle\langle t\rangle}$ then ( $\left.m, p\right) \cdot A \in$ $\mathcal{T}_{\langle s\rangle\langle t\rangle}$;

(4) for every $A \in \mathcal{L}_{2}$ and for every $\langle s\rangle,\langle t\rangle \in \mathcal{S}_{+}$such that $\langle s\rangle$ reg $\langle t\rangle$ and for every $m \in \mathbb{R} \backslash\{0\}$, if $A \in \mathcal{T}_{\langle s\rangle\langle t\rangle}$ then $(m, m) \cdot A \in \mathcal{T}_{\langle s\rangle\langle t\rangle}$;

(5) for every $\langle s\rangle \in \mathcal{S}_{0}$ there exists a set $A \in \mathcal{L}_{2}, A \in \mathcal{T}_{\langle s\rangle\langle s\rangle}$ such that for every $m \in \mathbb{R}$, $|m|<1$, the set $(m, m) \cdot A$ does not belong to $\mathcal{T}_{\langle s\rangle\langle s\rangle}$.

Proof. Properties (1)-(4) follow from the definition of the topology $\mathcal{T}_{\langle s\rangle\langle t\rangle}$ and Theorem 29 point (4). We give the proof only for (5).

Fix $\langle s\rangle \in \mathcal{S}_{0}$. Let $\left\{s_{n_{k}}\right\}_{k \in \mathbb{N}}$ be a subsequence of $\langle s\rangle$ such that

$$
\lim _{n \rightarrow \infty} \frac{s_{n_{k}}}{s_{n_{k}+1}}=0
$$

Let

$$
X:=\bigcup_{k=1}^{\infty}\left[\frac{1}{s_{n_{k}+1}}, \frac{1}{\sqrt{s_{n_{k}} s_{n_{k}+1}}}\right] .
$$

As was mentioned at the beginning, the one-dimensional version of a density topology with respect to a fixed sequence was considered in [1]. The definitions of the operator $\Phi_{\langle s\rangle}$ and the topology $\mathcal{T}_{\langle s\rangle}$ for the fixed sequence $\langle s\rangle$ are analogous to those in the two-dimensional case so we omit them.

The set $Y:=(\mathbb{R} \backslash X) \cup\{0\}$ belongs to $\mathcal{T}_{\langle s\rangle}$ (see [1, proof of Theorem 3]).

Define

$$
A:=\bigcup_{y \in Y \backslash \mathbb{R}_{-}}((\{-y, y\} \times[-y, y]) \cup([-y, y] \times\{-y, y\})) .
$$

An analysis similar to that in [3, proof of Theorem 2.6] shows that $A \in \mathcal{T}_{\langle s\rangle\langle s\rangle}$. 
For $m=0$ it is obvious that $(m, m) \cdot A \notin \mathcal{T}_{\langle s\rangle\langle s\rangle}$.

Without loss of generality we assume now that $m \in(0,1)$.

Let $k_{0}$ be a positive integer such that $\sqrt{s_{n_{k}} / s_{n_{k}+1}}<m$ for $k>k_{0}$. Then the set $m \cdot Y_{m}$, where

$$
Y_{m}:=\left(\mathbb{R} \backslash X_{m}\right) \cup\{0\} \quad \text { and } \quad X_{m}:=\bigcup_{k=k_{0}}^{\infty}\left[\frac{1}{s_{n_{k}+1}}, \frac{1}{\sqrt{s_{n_{k}} s_{n_{k}+1}}}\right],
$$

does not belong to $\mathcal{T}_{\langle s\rangle}$ (see [1, proof of Theorem 4]) and, again following ideas from [3, proof of Theorem 2.6], we get that the set

$$
(m, m) \cdot\left[\bigcup_{y \in Y_{m} \backslash \mathbb{R}_{-}}((\{-y, y\} \times[-y, y]) \cup([-y, y] \times\{-y, y\}))\right]
$$

does not belong to $\mathcal{T}_{\langle s\rangle\langle s\rangle}$, so neither does the set $(m, m) \cdot A$.

The next theorem expresses the connection between $\mathcal{T}_{\langle s\rangle\langle t\rangle}$ and the product topology $\mathcal{T}_{\langle s\rangle} \times \mathcal{T}_{\langle t\rangle}$.

THEOREM 31. For every $\langle s\rangle,\langle t\rangle \in \mathcal{S}$ the product topology $\mathcal{T}_{\langle s\rangle} \times \mathcal{T}_{\langle t\rangle}$ is contained in $\mathcal{T}_{\langle s\rangle\langle t\rangle}$.

PROOF. Let $\langle s\rangle,\langle t\rangle \in \mathcal{S}$ and let $E \in \mathcal{T}_{\langle s\rangle} \times \mathcal{T}_{\langle t\rangle}$. Fix any point $\left(x_{0}, y_{0}\right) \in E$ and a subsequence $\left\{n_{k}\right\}_{k \in \mathbb{N}}$ of the sequence of all natural numbers. Define $R_{k}:=$ $R\left(\left(x_{0}, y_{0}\right), 1 / s_{n_{k}}, 1 / t_{n_{k}}\right)$.

Since $E \in \mathcal{T}_{\langle s\rangle} \times \mathcal{T}_{\langle t\rangle}$, there exist sets $A, B \subset \mathbb{R}$, such that $A \times B \subset E, x_{0} \in A \subset$ $\Phi_{\langle s\rangle}(A)$ and $y_{0} \in B \subset \Phi_{\langle t\rangle}(B)$. Therefore for every $\epsilon>0$ there exists $k_{0} \in \mathbb{N}$ such that for every natural $k, k>k_{0}$,

$$
\frac{\lambda_{1}\left(\left(-1 / s_{n_{k}}, 1 / s_{n_{k}}\right) \backslash A\right)}{2 / s_{n_{k}}}<\frac{\epsilon}{2} \quad \text { and } \quad \frac{\lambda_{1}\left(\left(-1 / t_{n_{k}}, 1 / t_{n_{k}}\right) \backslash B\right)}{2 / s_{n_{k}}}<\frac{\epsilon}{2} .
$$

Since

$$
\begin{aligned}
P_{k} \backslash E \subset\left(\left(\left(-1 / s_{n_{k}}, 1 / s_{n_{k}}\right) \backslash A\right) \times\left(-1 / t_{n_{k}}, 1 / t_{n_{k}}\right)\right) \\
\cup\left(\left(-1 / s_{n_{k}}, 1 / s_{n_{k}}\right) \times\left(-1 / t_{n_{k}}, 1 / t_{n_{k}}\right) \backslash B\right),
\end{aligned}
$$

it follows that

$$
\lambda_{2}\left(R_{k} \backslash E\right) \leq \frac{\epsilon}{2} \cdot \frac{2}{s_{n_{k}}} \cdot \frac{2}{t_{n_{k}}}+\frac{2}{s_{n_{k}}} \cdot \frac{\epsilon}{2} \cdot \frac{2}{t_{n_{k}}}=\epsilon \cdot \lambda_{2}\left(R_{k}\right) .
$$

Hence $\left(x_{0}, y_{0}\right) \in \Phi_{\langle s\rangle\langle t\rangle}(E)$.

THEOREM 32. For every $\langle s\rangle \in \mathcal{S}_{0}$ and $\langle t\rangle \in \mathcal{S}$ the topologies $\mathcal{T}_{\langle s\rangle} \times \mathcal{T}_{\langle t\rangle}$ and $\mathcal{T}_{\langle s\rangle\langle t\rangle}$ are different. 
Proof. Let $\langle s\rangle \in \mathcal{S}_{0}$ and $\langle t\rangle \in \mathcal{S}$. Then $\mathcal{T}_{\langle s\rangle} \subset \mathcal{T}_{\langle t\rangle}$ (see [1]). Define $G:=\left(\mathbb{R}^{2} \backslash \Delta\right) \cup$ $\{(0,0)\}$, where $\Delta=\{(x, x): x \in \mathbb{R}\}$. Then $G \in \mathcal{T}_{\langle s\rangle\langle t\rangle}$ is a set of full measure. If $G \in$ $\mathcal{T}_{\langle s\rangle} \times \mathcal{T}_{\langle t\rangle}$ then there exist sets $A \in \mathcal{T}_{\langle s\rangle}$ and $B \in \mathcal{T}_{\langle t\rangle}$ such that $(0,0) \in A \times B \subset G$. Let $C:=A \cap B$. Then $C$ is nonempty and $C$ is open in $\mathcal{T}_{\langle t\rangle}$, so $C \backslash\{0\}$ cannot be empty. Then $(C \times C \backslash\{(0,0\}) \cap \Delta \neq \emptyset$, but $C \times C \subset G$, which is impossible, so $G \in \mathcal{T}_{\langle s\rangle\langle t\rangle} \backslash \mathcal{T}_{\langle s\rangle} \times \mathcal{T}_{\langle t\rangle}$.

For the sake of simplicity we have presented all results in $\mathbb{R}^{2}$ but they can be easily generalized to Euclidean spaces of dimension higher than two. In $\mathbb{R}^{m}$ we consider sets of $m$ sequences from the family $\mathcal{S}:\left\{\left\langle s^{p}\right\rangle: p \in\{1, \ldots, m\}\right\}$. For such a set we can define a density operator which for measurable set $A \subset \mathbb{R}^{m}$ is the set of all points $\left(x_{1}, \ldots, x_{m}\right)$ for which

$$
\lim _{n \rightarrow+\infty} \frac{\lambda_{m}\left(A \cap\left(\left(x_{1}-1 / s_{n}^{1}, x_{1}+1 / s_{n}^{1}\right) \times \cdots \times\left(x_{m}-1 / s_{n}^{m}, x_{m}+1 / s_{n}^{m}\right)\right)\right)}{2^{m} / s_{n}^{1} \cdots s_{n}^{m}}=1 .
$$

Following Saks (see [5]), a set of sequences $\left\{\left\langle s^{p}\right\rangle: p \in\{1, \ldots, m\}\right\}$ will be called regular if there exists a positive number $\alpha$ such that

$$
\frac{\min _{p \in\{1, \ldots, m\}} s_{n}^{p}}{\max _{p \in\{1, \ldots, m\}} s_{n}^{p}}>\alpha \quad \text { for } n \in \mathbb{N} .
$$

Having defined these notions we can prove theorems analogous to the two-dimensional case. The same line of reasoning applies to higher-dimensional versions.

\section{References}

[1] M. Filipczak and J. Hejduk, 'On topologies associated with the Lebesgue measure', Tatra Mt. Math. Publ. 28 (2004), 187-197.

[2] J. Hejduk and A. Loranty, 'On lower and semi-lower density operators', Georgian Math. J. 17(4) (2007), 661-671.

[3] G. Horbaczewska and E. Wagner-Bojakowska, 'Some modifications of density topologies', J. Appl. Anal. 7(1) (2001), 91-105.

[4] L. Lukás, J. Maly̌ and L. Zajiček, Fine Topology Methods in Real Analysis and Potential Theory, Lecture Notes in Mathematics, 1189 (Springer, Berlin, 1986).

[5] S. Saks, Theory of the Integral, Monografie Matematyczne, VII (Stechert, New York 1937).

[6] W. Wilczyński, Density Topologies, (ed. E. Pap) (North Holland, Amsterdam, 2002), Chapter 15 in Handbook of Measure Theory, pp. 675-702.

GRAŻYNA HORBACZEWSKA, Department of Mathematics and Computer Science, University of Łódz, Banacha 22, 90238 Łódz, Poland

e-mail: grhorb@math.uni.lodz.pl 